\title{
Wealth Effects on Self-insurance
}

\author{
Kangoh Lee \\ Department of Economics, San Diego State University, 5500 Campanile Drive, San Diego, \\ CA 92182-4485, U.S.A. \\ E-mail:klee@mail.sdsu.edu
}

This paper considers the wealth effects on self-insurance investment that reduces loss. Wealthier individuals can bear the risk better, and invest less in self-insurance with two states of the world. Self-insurance, like insurance, is thus an inferior good. This known result does not extend to many states. The reason is that an increase in self-insurance does not necessarily reduce final wealth in good states and increase it in bad states. Self-insurance thus may not act as insurance, and wealthier individuals may not necessarily invest less in self-insurance. The paper proposes a condition under which self-insurance is inferior, and a condition under which it is normal.

The Geneva Risk and Insurance Review (2010) 35, 160-171. doi:10.1057/grir.2010.6; published online 24 August 2010

Keywords: self-insurance; inferior; normal

\section{Introduction}

One of the most important results in the literature on insurance economics states that insurance is an inferior good under decreasing absolute risk aversion (DARA) (Mossin, 1968; Schlesinger, 2000). However, it has not been studied whether self-insurance is an inferior good with multiple states under DARA. The reason for this omission in the literature may be that selfinsurance is a type of insurance, and it is believed that the general results on insurance should carry over to self-insurance. This belief is not correct, and the omission is unfortunate because self-insurance has been extensively modelled in various fields of economics since the inception of the concept by Ehrlich and Becker (1972), and because self-insurance such as fire extinguishers and medical checkups has been widely used in practice. This paper studies the wealth effects on self-insurance with multiple states of the world, and demonstrates that self-insurance may be either a normal good or an inferior good under DARA.

The known result that insurance is inferior under DARA is intuitive. With an unfair premium, risk-averse individuals purchase a partial coverage of insurance, exposing them to random loss. As wealthier individuals are more 
willing to bear risk with DARA (Arrow, 1970), they purchase less insurance, making insurance inferior. ${ }^{1}$

The same argument applies to self-insurance with two states, either a loss (the bad state) or no loss (the good state) occurring. In the good state with no loss, an increase in self-insurance does not benefit, but increases the cost of self-insurance, decreasing final random wealth. In the bad state, an increase in self-insurance reduces the loss more than increases the cost of self-insurance, increasing final random wealth. Self-insurance thus acts as insurance, and selfinsurance is inferior under DARA. However, this two-state result does not carry over to many states. The difficulty is that self-insurance does not necessarily reduce larger losses in the bad states more effectively than smaller losses in the good states. Rather, the effectiveness of a given self-insurance investment across different states depends on its technologies and the nature of the losses. Self-insurance thus may not act as insurance, and wealthier individuals may invest less or more in self-insurance. The analysis establishes some sufficient conditions under which self-insurance is inferior, and some conditions under which it is normal.

A few papers have considered the effects of increased risk aversion on selfinsurance (Dionne and Eeckhoudt, 1985; Briys and Schlesinger, 1990; Lee, 1998; Dachraoui et al., 2004). Sweeney and Beard (1992) and Lee (2005) analyze the wealth effects on self-protection, and on self-insurance, respectively, in a two-state model. But the wealth effects on self-insurance with multiple states have not been studied.

The paper is organized as follows. The next section models self-insurance. The subsequent section considers the difference between insurance and selfinsurance. The next following section discusses the case with two loss states. The penultimate section analyzes many states, and the last section offers a conclusion.

\section{The model}

A risk-averse individual has initial wealth $w_{o}$. The individual may lose part or all of her wealth. The size of loss in state $\theta$, given self-insurance activity $x$, is written as $\ell(x, \theta) . \theta$ is a random variable, and distributed according to the distribution function $F(\theta)$ on the support $[\underline{\theta}, \bar{\theta}]$ with $f(\theta)=F^{\prime}(\theta)$. Without loss of generality, assume

$$
\ell_{\theta}(x, \theta) \equiv \frac{\partial \ell(x, \theta)}{\partial \theta}>0,
$$

\footnotetext{
${ }^{1}$ A referee provided this intuition.
} 
meaning that a state with a larger $\theta$ represents a worse (or less favourable or lucky) state with a larger loss, given self-insurance investment $x$. To reduce the loss $\ell(x, \theta)$, the individual invests in $x$. The cost of $x$ is $c(x)$, an increasing and convex function. An increase in self-insurance decreases the loss, and $\ell_{x}(x, \theta) \equiv \partial \ell(x, \theta) / \partial x \leqslant 0$ with strict inequality for some $\theta$, and assume $\ell_{x x}(x, \theta) \geqslant 0$ for an interior solution later. Final wealth in state $\theta$ then reads as $w(x, \theta) \equiv w_{o}-\ell(x, \theta)-c(x)$. Since a larger $\theta$ means a larger loss,

$$
w_{\theta}(x, \theta)=-\ell_{\theta}(x, \theta)<0 .
$$

The individual's problem is to choose $x$ to maximize her expected utility of wealth $E U \equiv \int_{\theta}^{\theta} U(w(x, \theta)) f(\theta) d \theta$, where $U^{\prime}>0$ and $U^{\prime \prime}<0$. The first-order condition for an interior maximum of $E U$ is

$$
\frac{d E U}{d x}=\int_{\underline{\theta}}^{\bar{\theta}} U^{\prime}(w(x, \theta)) w_{x}(x, \theta) f(\theta) d \theta=0 .
$$

For the condition to hold, the $w_{x}(x, \theta)$ terms must be positive for some $\theta$ s, and must be negative for other $\theta$ s. That is, in some states, an increase in selfinsurance must increase final wealth by decreasing the loss more than increasing the cost, because $w_{x}(x, \theta)=-\ell_{x}(x, \theta)-c^{\prime}(x)>0$ when $-\ell_{x}()>.c^{\prime}(x)$. Analogously, in other states, $-\ell_{x}()<.c^{\prime}(x)$, and $w_{x}()<$.0 . Interpreting $U^{\prime}(w().) w_{x}()>$.0 as the marginal benefit from an increase in self-insurance while interpreting $U^{\prime}(w().) w_{x}()<$.0 as the marginal cost, condition (3) states that at an optimum, the marginal benefits over some states must equal the marginal costs over other states. Since $U($.$) is concave while c($.$) and \ell($.) are convex,

$$
\begin{aligned}
D \equiv \frac{d^{2} E U}{d x^{2}}=\int_{\underline{\theta}}^{\bar{\theta}}\left[U^{\prime \prime}(w(x, \theta))\left(w_{x}(x, \theta)\right)^{2}\right. \\
\left.+U^{\prime}(w(x, \theta)) w_{x x}(x, \theta)\right] f(\theta) d \theta<0,
\end{aligned}
$$

because $w_{x x}()=.-\ell_{x x}()-.c^{\prime \prime}() \leqslant$.0 . The second-order condition is thus satisfied.

The goal of the analysis is to determine the effect of an increase in the initial wealth, $w_{o}$, on the demand for self-insurance, $x$. To this end, the first-order condition in (3) is totally differentiated to obtain

$$
\frac{\partial x}{\partial w_{o}}=-\frac{1}{D} \int_{\underline{\theta}}^{\bar{\theta}} U^{\prime \prime}(w(x, \theta)) w_{x}(x, \theta) f(\theta) d \theta,
$$

where use is made of $\partial w(x, \theta) / \partial w_{o}=1$. The sign of (4) cannot be unambiguously determined, because $w_{x}()>$.0 for some $\theta$ s while $w_{x}()<$.0 for other $\theta$ s, as noted 
in the discussion of (3). It depends in part on the individual's preferences toward risk. Letting $A(w(x, \theta)) \equiv-U^{\prime \prime}(w(x, \theta)) / U^{\prime}(w(x, \theta))$ denote the coefficient of absolute risk aversion, (4) is rewritten as

$$
\frac{\partial x}{\partial w_{o}}=-\frac{1}{D} \int_{\underline{\theta}}^{\bar{\theta}}-A(w(x, \theta)) U^{\prime}(w(x, \theta)) w_{x}(x, \theta) f(\theta) d \theta .
$$

Before studying (5), it proves useful to discuss the difference between wealth effects on self-insurance and those on insurance, as the difference provides an insight into the issue at hand.

\section{Self-insurance, insurance and a single sign change}

Consider a standard model of the demand for insurance. The loss in state $\theta$ is simply $\ell(\theta)$, because insurance does not reduce the loss. Assume that an individual buys an insurance coverage $\alpha \ell(\theta)$ with $\alpha \in[0,1]$ for a premium of $\alpha \pi$. There is a loading factor $\beta \geqslant 1$, and $\pi=\beta E[\ell(\theta)]=\beta \int_{\theta}^{\theta} \ell(\theta) f(\theta) d \theta$. Final wealth in each state $\theta$ reads as $w(\alpha, \theta)=w_{o}-\ell(\theta)+\alpha(\ell(\theta)-\pi)$, and the expected utility is written as $E V=\int_{\underline{\theta}}^{\theta} U(w(\alpha, \theta)) f(\theta) d \theta$. The first-order condition for an optimal $\alpha$ is

$$
\frac{d E V}{d \alpha}=\int_{\underline{\theta}}^{\bar{\theta}} U^{\prime}(w(\alpha, \theta)) w_{\alpha}(\alpha, \theta) f(\theta) d \theta=0 .
$$

At an interior optimal $\alpha$, it must be that $w_{\alpha}(\alpha, \theta) \equiv \ell(\theta)-\pi<0$ for some $\theta$ s while it is positive for other $\theta$ s. Since $\ell(\theta)$ increases with $\theta$ from (1), and since $\pi$ is independent of $\theta$, there exists a critical value, $\hat{\theta} \in(\underline{\theta}, \bar{\theta})$, such that

$$
w_{\alpha}(\alpha, \theta) \equiv \ell(\theta)-\pi<(=,>) 0 \text { for } \theta<(=,>) \hat{\theta}
$$

The states with $\theta<\hat{\theta}$ are the good states while the states with $\theta \geqslant \hat{\theta}$ are the bad states. The first-order condition (6) then states that at an optimal $\alpha$, the marginal costs of an increase in $\alpha$ over the good states must equal the marginal benefits over the bad states. 
The wealth effect on insurance reads as

$$
\begin{aligned}
\frac{\partial \alpha}{\partial w_{o}}= & -\frac{1}{H} \int_{\underline{\theta}}^{\bar{\theta}} U^{\prime \prime}(w(\alpha, \theta)) w_{\alpha}(\alpha, \theta) f(\theta) d \theta \\
= & \frac{1}{H} \int_{\underline{\theta}}^{\bar{\theta}} A(w(\alpha, \theta)) U^{\prime}(w(\alpha, \theta)) w_{\alpha}(\alpha, \theta) f(\theta) d \theta \\
= & \frac{1}{H}\left[\int_{\underline{\theta}}^{\hat{\theta}} A(w(\alpha, \theta)) U^{\prime}(w(\alpha, \theta)) w_{\alpha}(\alpha, \theta) f(\theta) d \theta\right. \\
& \left.+\int_{\hat{\theta}}^{\bar{\theta}} A(w(\alpha, \theta)) U^{\prime}(w(\alpha, \theta)) w_{\alpha}(\alpha, \theta) f(\theta) d \theta\right]<0,
\end{aligned}
$$

under DARA, where $H=\int_{\theta}^{\bar{\theta}} U^{\prime \prime}(w(\alpha, \theta))\left[w_{\alpha}(\alpha, \theta)\right]^{2} f(\theta) d \theta<0$. To see (8), note that the first integral inside squared brackets is negative because of (7) while the second one is positive. With DARA, $A(w(\alpha, \theta))$ s in the first integral are smaller than $A(w(\alpha, \theta))$ s in the second one. The first integral is thus outweighed by the second one, making the terms inside the squared brackets positive, because those terms would be zero by (6) if $A(w(x, \theta))$ s were the same. Considering that $H<0,(8)$ is negative, and insurance is inferior. Intuitively, due to diminishing marginal utility of wealth, an increase in $w_{o}$ decreases both the marginal costs of an increase in $\alpha$ over the good states with $\theta<\hat{\theta}$ and the marginal benefits over the bad states with $\theta>\hat{\theta}$. Under DARA, an increase in $w_{o}$ decreases the marginal benefits in the bad states more than the marginal costs in the good states, decreasing $\alpha$. Under increasing absolute risk aversion (IARA), the opposite holds, and $\partial \alpha / \partial w_{o}>0$, and insurance is normal. ${ }^{2}$

The wealth effects on self-insurance depend on the sign of $w_{x}(x, \theta)$ in (5), and those on insurance depend on the sign of $w_{\alpha}(\alpha, \theta)$ in (6). The key difference is that there is no clear relationship between the sign of $w_{x}(x, \theta)$ and $\theta$, but $w_{\alpha}(\alpha, \theta)$ changes its sign once from minus to plus, as in (7). This is the singlecrossing condition in Diamond and Stiglitz (1974) under which more riskaverse individuals increase the level of the control variable. Thus, under DARA, wealthier individuals purchase less insurance, so insurance is inferior. Intuitively, with an unfair premium due to loading, risk-averse individuals

${ }^{2}$ Since DARA is considered a natural assumption, and since the IARA case is analogous, the subsequent analysis focuses mainly on the DARA case to conserve space. 
purchase partial coverage and, with DARA, wealthier individuals are less risk averse and therefore purchase less insurance coverage.

Turning to self-insurance, self-insurance would be inferior with DARA if $w_{x}(x, \theta)$ changed its sign once from minus to plus in a manner analogous to (7). Observe that this single-crossing condition means that self-insurance is more productive at large losses. However, there is no a priori reason that this condition holds, because the productivity of self-insurance depends on the self-insurance technologies and the nature of the losses. For example, the design and construction of a building to reduce the loss resulting from an earthquake may be quite effective in case of a small earthquake, as the design enables the building to withstand a certain level of earthquakegenerated force. A strong earthquake, however, may destroy all buildings, and make such design and construction almost useless. More importantly, among many earthquakes other than a small one or a strong one, the design and construction may be more effective or less effective in reducing the loss from one earthquake than from another, and the effectiveness does not necessarily increase with the strength of earthquake. The single-crossing from negative to positive thus may not hold, and self-insurance may not be inferior with DARA.

The discussion above indicates that self-insurance is normal with DARA if the single-crossing condition is reversed, so that $w_{x}(x, \theta)$ changes its sign once from plus to minus. In order for this to occur, there must be no state in which the loss equals zero. The reason is that, in a no-loss state, $\ell(x, \theta)=0$ for all $x$, implying that $\ell_{x}(x, \theta)=0$, in which case $w_{x}(x, \theta)=-\ell_{x}(x, \theta)-c^{\prime}(x)=-c^{\prime}(x)$ is negative rather than positive. As a rationale for the absence of a state with no loss, an example of medical expenses may be considered. $\underline{\theta}$ could represent a state in which the individual must meet usual medical expenses, with higher values of $\theta$ representing states in which these plus some additional, unusual medical expenses must be covered. Since the absence of the no-loss state and the reversal of the single-crossing condition can be best illustrated with two loss states, the next section considers two loss states, and the following section generalizes the results to many states.

\section{Two loss states}

Assume that there are only two states, $\theta_{1}$ and $\theta_{2}$ with $\theta_{1}<\theta_{2}$, and that losses occur in both states. The first-order condition for an optimal self-insurance $x,(3)$, becomes

$$
\frac{d E U}{d x}=p_{1} U^{\prime}\left(w\left(x, \theta_{1}\right)\right) w_{x}\left(x, \theta_{1}\right)+p_{2} U^{\prime}\left(w\left(x, \theta_{2}\right)\right) w_{x}\left(x, \theta_{2}\right)=0,
$$


166

where $p_{i}=f\left(\theta_{i}\right), i=1,2$. The comparative static result (4) becomes

$$
\frac{\partial x}{\partial w_{o}}=-\frac{1}{D}\left[p_{1} U^{\prime \prime}\left(w\left(x, \theta_{1}\right)\right) w_{x}\left(x, \theta_{1}\right)+p_{2} U^{\prime \prime}\left(w\left(x, \theta_{2}\right)\right) w_{x}\left(x, \theta_{2}\right)\right],
$$

where $D<0$. Thus, $\partial x / \partial w_{o}$ has the same sign as

$$
\begin{aligned}
& -p_{1} A\left(w\left(x, \theta_{1}\right)\right) U^{\prime}\left(w\left(x, \theta_{1}\right)\right) w_{x}\left(x, \theta_{1}\right) \\
& -p_{2} A\left(w\left(x, \theta_{2}\right)\right) U^{\prime}\left(w\left(x, \theta_{2}\right)\right) w_{x}\left(x, \theta_{2}\right) \\
= & p_{2} A\left(w\left(x, \theta_{1}\right)\right) U^{\prime}\left(w\left(x, \theta_{2}\right)\right) w_{x}\left(x, \theta_{2}\right) \\
& -p_{2} A\left(w\left(x, \theta_{2}\right)\right) U^{\prime}\left(w\left(x, \theta_{2}\right)\right) w_{x}\left(x, \theta_{2}\right) \\
= & {\left[A\left(w\left(x, \theta_{1}\right)\right)-A\left(w\left(x, \theta_{2}\right)\right)\right] p_{2} U^{\prime}\left(w\left(x, \theta_{2}\right)\right) w_{x}\left(x, \theta_{2}\right) . }
\end{aligned}
$$

The first equality uses the first-order condition (9).

With DARA, $A\left(w\left(x, \theta_{1}\right)\right)<A\left(w\left(x, \theta_{2}\right)\right)$, and the expression in brackets is negative. If the single-crossing condition $w_{x}\left(x, \theta_{1}\right)<0$ and $w_{x}\left(x, \theta_{2}\right)>0$ holds, then $\partial x / \partial w_{o}<0$ and self-insurance is inferior. If the reverse inequalities $w_{x}\left(x, \theta_{1}\right)>0$ and $w_{x}\left(x, \theta_{2}\right)<0$ hold, then $\partial x / \partial w_{o}>0$ and self-insurance is normal. This discussion illustrates that self-insurance can be normal even with DARA. ${ }^{3}$

\section{Many states}

Returning to the analysis of many states, consider first the case with a singlecrossing state, so that $w_{x}(x, \theta)$ changes its sign once at $\theta^{*} \in(\underline{\theta}, \bar{\theta})$ with $\theta^{*}$ denoting the crossing state. The states, $\theta \in[\underline{\theta}, \bar{\theta}]$, are then divided into two groups, the first group of good states with $\theta \leqslant \theta^{*}$ and the second group of bad states with $\theta>\theta^{*}$. Suppose that $w_{x}(x, \theta) \leqslant(>) 0$ for $\theta \leqslant(>) \theta^{*}$ with the equality holding only at $\theta^{*}$, so that an increase in self-insurance decreases random final wealth in all good states and increases it in all bad states. As discussed in 'Selfinsurance, insurance and a single sign change' section, this single-crossing condition ensures that more risk-averse individuals desire more self-insurance and hence wealthier individuals with DARA choose less self-insurance, making self-insurance inferior.

At the critical $\theta, \theta^{*}, w_{x}\left(x, \theta^{*}\right)=0$, and $\theta^{*}$ may depend on $x$. Since $x$ is the choice of self-insurance that maximizes the expected utility of an individual under consideration, $\theta^{*}$ depends on each individual decision maker. In order

\footnotetext{
${ }^{3}$ With IARA, self-insurance would be normal (inferior) if $w_{x}\left(x, \theta_{2}\right)<(>) 0$.
} 
for the single-crossing condition to apply generally, the condition must hold for all feasible $x$. In addition, normality or inferiority of self-insurance depends on the nature of losses such as absence of a no-loss state and on self-insurance technology, as noted earlier. The critical $\theta$, denoted $\theta^{* *}$, in a reversal of the single-crossing condition for normality of self-insurance may then differ from $\theta^{*}$ for inferiority of self-insurance. Thus, if there is no no-loss state, and if $w_{x}(x, \theta) \geqslant(<) 0$ for $\theta \leqslant(>) \theta^{* *}(x)$, self-insurance is normal. This result may be stated as:

Proposition 1 Assume DARA. (i) A sufficient condition for self-insurance to be inferior is that there exists $\theta^{*}(x)$ for all feasible $x$ such that $w_{x}(x, \theta) \leqslant(>) 0$ for $\theta \leqslant(>) \theta^{*}(x)$ (ii) Sufficient conditions for self-insurance to be normal are that there is no no-loss state and there exists $\theta^{* *}(x)$ for all feasible $x$ such that $w_{x}(x, \theta) \geqslant(<) 0$ for $\theta \leqslant(>) \theta^{* *}(x)$.

To see (i) of the proposition, observe that $\partial x / \partial w_{o}$ in (5) has the same sign as

$$
\begin{aligned}
& -\int_{\underline{\theta}}^{\bar{\theta}} A(w(x, \theta)) U^{\prime}(w(x, \theta)) w_{x}(x, \theta) f(\theta) d \theta \\
= & -\int_{\underline{\theta}}^{\theta^{*}} A(w(x, \theta)) U^{\prime}(w(x, \theta)) w_{x}(x, \theta) f(\theta) d \theta \\
& -\int_{\theta^{*}} A(w(x, \theta)) U^{\prime}(w(x, \theta)) w_{x}(x, \theta) f(\theta) d \theta \\
< & -A\left(w\left(x, \theta^{*}\right)\right) \int_{\underline{\theta}}^{\theta^{*}} U^{\prime}(w(x, \theta)) w_{x}(x, \theta) f(\theta) d \theta \\
& -A\left(w\left(x, \theta^{*}\right)\right) \int_{\theta^{*}}^{\bar{\theta}} U^{\prime}(w(x, \theta)) w_{x}(x, \theta) f(\theta) d \theta \\
= & -A\left(w\left(x, \theta^{*}\right)\right) \int_{\underline{\theta}}^{\bar{\theta}} U^{\prime}(w(x, \theta)) w_{x}(x, \theta) f(\theta) d \theta=0 \text { under DARA, }
\end{aligned}
$$

where $\theta^{*}=\theta^{*}(x)$. The inequality follows because $A(w(x, \theta))<A\left(w\left(x, \theta^{*}(x)\right)\right)$ and $w_{x}(x, \theta) \leqslant 0$ for $\theta \leqslant \theta^{*}(x)$, while the reverse inequalities hold for $\theta>\theta^{*}(x)$. The last equality comes from the first-order condition (3). Under the condition in 
(ii), the inequality in (11) is reversed with $\theta^{* *}$ replacing $\theta^{*}$ and self-insurance is normal.

The proposition shows that self-insurance can be normal even under DARA, as in (ii). Thus, DARA is neither sufficient for nor necessary to inferiority of self-insurance. ${ }^{4}$

While the proposition concerns the single-crossing case, multiple crossings can occur. For expositional simplicity, the analysis is restricted to two crossings although it extends to many crossings. ${ }^{5}$ Let $\theta_{1}^{*}$ and $\theta_{2}^{*}$ denote the two crossings with $\underline{\theta}<\theta_{1}^{*}<\theta_{2}^{*}<\bar{\theta}$. Define $b_{i}$ as

$$
\begin{aligned}
& b_{1} \equiv \int_{\underline{\theta}}^{\theta_{1}^{*}} U^{\prime}(.) w_{x}(x, \theta) f(\theta) d \theta, b_{2} \equiv \int_{\theta_{1}^{*}}^{\theta_{2}^{*}} U^{\prime}(.) w_{x}(x, \theta) f(\theta) d \theta, \\
& b_{3} \equiv \int_{\theta_{2}^{*}}^{\bar{\theta}} U^{\prime}(.) w_{x}(x, \theta) f(\theta) d \theta .
\end{aligned}
$$

In addition, define $B_{i}$ as

$$
B_{1}=b_{1}, B_{2}=b_{1}+b_{2}, B_{3}=b_{1}+b_{2}+b_{3} .
$$

Observe that $B_{3}=0$ given the first-order condition (3). Using the definition of $B_{i}$, The following result then can be stated:

Proposition 2 Assume DARA. (i) Sufficient conditions for self-insurance to be inferior are that there exist $\theta_{1}^{*}(x)$ and $\theta_{2}^{*}(x)$ for all feasible $x$ such that $B_{i} \leqslant 0$ for all $i=1,2,3$. (ii) Sufficient conditions for self-insurance to be normal are that there is no no-loss state and there exist $\theta_{1}^{* *}(x)$ and $\theta_{2}^{* *}(x)$ for all feasible $x$ such that $B_{i} \geqslant 0$ for all $i=1,2,3$.

To see (i), observe that $w_{x}(x, \theta)$ has the same sign for all $\theta \in\left[\underline{\theta}, \theta_{1}^{*}(x)\right]$ by the definition of the first crossing point $\theta_{1}^{*}(x)$. In addition, $B_{1}=b_{1} \leqslant 0$ by assumption in (i). Thus,

$$
w_{x}(x, \theta) \leqslant 0 \text { for } \theta \in\left[\underline{\theta}, \theta_{1}^{*}(x)\right] .
$$

By the definition of the second crossing point, $\theta_{2}^{*}(x)$,

\footnotetext{
${ }^{4}$ By an analogous argument, it can be shown that with IARA, self-insurance is normal under the condition in (i) and inferior under the condition in (ii). Thus, IARA is neither necessary to nor sufficient for normality of self-insurance.

${ }^{5}$ An earlier version considered a general case with multiple crossings.
} 


$$
w_{x}(x, \theta) \geqslant 0 \text { for } \theta \in\left[\theta_{1}^{*}(x), \theta_{2}^{*}(x)\right] \text {, and } w_{x}(x, \theta) \leqslant 0 \text { for } \theta \in\left[\theta_{2}^{*}(x), \bar{\theta}\right] \text {. }
$$

Case (i) thus concerns the situation in which $w_{x}(x, \theta)$ changes its sign twice from minus to plus to minus. Now, $\partial x / \partial w_{o}$ in (5) has the same sign as

$$
\begin{aligned}
& -\int_{\theta}^{\bar{\theta}} A(w(x, \theta)) U^{\prime}(w(x, \theta)) w_{x}(x, \theta) f(\theta) d \theta \\
= & -\int_{\underline{\theta}}^{\theta_{1}^{*}} A(w(x, \theta)) U^{\prime}(w(x, \theta)) w_{x}(x, \theta) f(\theta) d \theta \\
& -\int_{\theta_{1}^{*}}^{\theta_{2}^{*}} A(w(x, \theta)) U^{\prime}(w(x, \theta)) w_{x}(x, \theta) f(\theta) d \theta \\
& -\int_{\theta_{2}^{*}} A(w(x, \theta)) U^{\prime}(w(x, \theta)) w_{x}(x, \theta) f(\theta) d \theta \\
< & -A\left(w\left(x, \theta_{1}^{*}\right)\right) b_{1}-A\left(w\left(x, \theta_{1}^{*}\right)\right) b_{2}-A(w(x, \bar{\theta})) b_{3} \\
= & -A\left(w\left(x, \theta_{1}^{*}\right)\right) B_{2}-A(w(x, \bar{\theta}))\left(B_{3}-B_{2}\right) \\
= & {\left[A(w(x, \bar{\theta}))-A\left(w\left(x, \theta_{1}^{*}\right)\right)\right] B_{2} \leqslant 0 \text { under DARA. } }
\end{aligned}
$$

The first inequality uses (12) and (13) with DARA. The next three equalities follow from the definition of $b_{i}$ and $B_{i}$. The last equality follows because $B_{3}=b_{1}+b_{2}+b_{3}=0$ in (3), and the assumption $B_{i} \leqslant 0$.

To see the intuition, consider $\theta \in\left[\theta_{1}^{*}(x), \bar{\theta}\right]=\left[\theta_{1}^{*}(x), \theta_{2}^{*}(x)\right] \cup\left[\theta_{2}^{*}(x), \bar{\theta}\right]$. Although $w_{x}(x, \theta) \geqslant 0$ for $\theta \in\left[\theta_{1}^{*}(x), \theta_{2}^{*}(x)\right]$ while $w_{x}(x, \theta) \leqslant 0$ for $\theta \in\left[\theta_{2}^{*}(x), \bar{\theta}\right]$ from (13), the average value of $w_{x}(x, \theta)$, weighted by $A(w(x, \theta))$, in $\left[\theta_{1}^{*}(x), \bar{\theta}\right]$ must be positive, because $B_{3}=0$ by (3) while $b_{1} \leqslant 0$ from (12). That is, roughly speaking, $b_{2}+b_{3} \geqslant 0$, given that $B_{3}=b_{1}+b_{2}+b_{3}=0$ and $b_{1} \leqslant 0$. Since states $\theta$ in $b_{1}$ represent the good states while states $\theta$ in $b_{2}$ and $b_{3}$ represent the bad states, an increase in self-insurance decreases final wealth in the good states while increasing it in the bad states. Self-insurance thus acts as insurance, and is inferior.

Part (ii) concerns the opposite case where $w_{x}(x, \theta)$ changes its sign twice from plus to minus to plus. Using the steps parallel to those used to establish 
(i) of Proposition 2, it is straightforward to establish that (ii) is sufficient for self-insurance to be normal.

\section{Conclusion}

An increase in self-insurance does not necessarily increase final wealth in the bad states or decrease final wealth in the good states, and self-insurance may not act as insurance. As a consequence, self-insurance is not necessarily inferior even under DARA. The analysis has proposed some sufficient conditions that determine the wealth effects on self-insurance. These conditions are restrictive, but may serve as a useful step toward less restrictive conditions future research may establish.

While the analysis has focused on the wealth effects on self-insurance, it has a general implication for economics of uncertainty and insurance beyond the wealth effects. Two-state models have been extensively used to analyze various economic questions under uncertainty because of their simplicity and intuitiveness. However, as this paper has shown in the case of the wealth effects on self-insurance, the two-state analysis may not extend to many states in other areas of uncertainty. For example, according to the standard two-state model, it may be thought that both insurance and self-insurance are inferior, which turns out to be an incorrect claim, as the analysis has demonstrated. By contrast, the two-state model may lead one to conclude that self-insurance and self-protection are fundamentally different activities, because selfinsurance is inferior while the wealth effect on self-protection is ambiguous (Sweeney and Beard, 1992). Nevertheless, with multiple states the wealth effects are ambiguous for both of these activities.

\section{Acknowledgements}

I am grateful to Georges Dionne for his comments, and to an anonymous referee who provided detailed and useful comments that improve the paper significantly.

\section{References}

Arrow, K. (1970) Essays in the Theory of Risk-bearing, Amsterdam: North Holland.

Briys, E. and Schlesinger, H. (1990) 'Risk aversion and the propensities for self-insurance and self-protection', Southern Economic Journal 57: 458-467.

Dachraoui, K., Dionne, G., Eeckhoudt, L. and Godfroid, P. (2004) 'Comparative mixed risk aversion: Definition and application to self-protection and willingness to pay', Journal of Risk and Uncertainty 29: 261-276.

Diamond, P. and Stiglitz, J. (1974) 'Increases in risk and in risk aversion', Journal of Economic Theory 8: $337-360$. 
Dionne, G. and Eeckhoudt, L. (1985) 'Self-insurance, self-protection and increased risk aversion', Economics Letters 17: 39-42.

Ehrlich, I. and Becker, G. (1972) 'Market insurance, self-insurance, and self-protection', Journal of Political Economy 90: 623-648.

Lee, K. (1998) 'Risk aversion and self-insurance-cum-protection', Journal of Risk and Uncertainty 17: $139-150$.

Lee, K. (2005) 'Wealth effects on self-insurance and self-protection against monetary and nonmonetary losses', Geneva Risk and Insurance Review 30: 147-159.

Mossin, J. (1968) 'Aspects of rational insurance purchasing', Journal of Political Economy 76: $552-568$.

Schlesinger, H. (2000) 'The theory of insurance demand', in G. Dionne (ed) Handbook of Insurance, Boston: Kluwer Academic Publishers, pp. 131-151.

Sweeney, G. and Beard, R. (1992) 'The comparative statics of self-protection', Journal of Risk and Insurance 59: 301-309.

\section{About the Author}

Kangoh Lee is a professor of Economics at San Diego State University. His fields of study include Public Economics, and Risk and Uncertainty. 